\title{
Baseline anemia and anemia grade are independent prognostic factors for stage IV non-small cell lung cancer
}

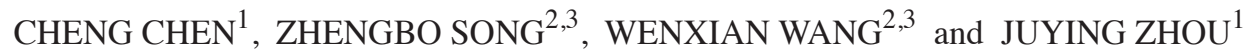 \\ ${ }^{1}$ Department of Radiotherapy, The First Affiliated Hospital of Soochow University, Suzhou, Jiangsu 215006; \\ ${ }^{2}$ Department of Chemotherapy, Zhejiang Cancer Hospital; ${ }^{3}$ Key Laboratory Diagnosis and \\ Treatment Technology on Thoracic Oncology, Hangzhou, Zhejiang 310022, P.R. China
}

Received July 18, 2020; Accepted January 11, 2021

DOI: $10.3892 /$ mco.2021.2221

\begin{abstract}
The current study aimed to investigate baseline anemia in patients with stage IV non-small cell lung cancer (NSCLC) and its relationship with clinicopathological features and prognosis. The clinical data of 4,874 patients with stage IV NSCLC were analyzed. The incidence of baseline anemia was observed. The relationship between baseline anemia and clinicopathological features was analyzed. Kaplan-Meier method and multivariate COX regression model were used to analyze the relationship of baseline anemia and prognosis of patients with NSCLC. Anemia classification was based on the criteria established by the National Cancer Institute (NCI). The mean hemoglobin $(\mathrm{Hb})$ was $123.32 \pm 20.31 \mathrm{~g} / \mathrm{l}$ in patients with stage IV NSCLC. The prevalence of baseline anemia was $32.09 \%$, among which $19.08,10.79,1.91$ and $0.31 \%$ had mild, moderate, severe, and life-threatening anemia, respectively. The prevalence of baseline anemia was higher in patients who were $>60$ years old, male, had smoking history, exhibited squamous cell carcinoma and bone metastasis, and the difference was statistically significant. Univariate analysis indicated that patients without anemia had longer overall survival (OS) compared with patients with baseline anemia (median OS: 28.0 months vs. 17.4 months, $\mathrm{P}<0.001)$. As the grade of anemia rises, it was indicated that OS became shorter. Patients with anemia grade 0 had the longest OS (median OS: 28.0 months), followed by patients with anemia grades 1 and 2 (median OS: 17.5 months). The patients with anemia grades 3 and 4 had the shortest OS (median OS: 8.6 months; $\mathrm{P}<0.001)$. Multivariate analysis demonstrated that baseline anemia and anemia grade were independent prognostic factors in patients with stage IV NSCLC. In conclusion, baseline anemia and anemia grade
\end{abstract}

Correspondence to: Dr Juying Zhou, Department of Radiotherapy, The First Affiliated Hospital of Soochow University, 188 Shizi Street, Suzhou, Jiangsu 215006, P.R. China

E-mail: zhoujuyingsy@163.com

Key words: baseline anemia, anemia grade, prognosis, non-small cell lung cancer are independent prognostic factors in patients with stage IV NSCLC.

\section{Introduction}

Lung cancer has the highest morbidity and mortality in China (1); 80-85\% of patients with lung cancer had non-small cell lung cancer (NSCLC), and the majority were advanced at the time of initial diagnosis. Tumor-associated anemia is one of the common concomitant diseases of malignant tumors (2). Tumor-associated anemia production can be caused by a variety of factors, including tumor factors or tumor treatment factors. Tumor-associated anemia is common in patients with lung cancer and is often dramatically increased due to cancer therapy, especially platinum chemotherapy (3-7). Tumor-associated anemia can cause a variety of clinical symptoms, seriously reduces the quality of life of patients, affects the patient's tolerance to treatment, and reduces tumor tissue sensitivity to treatment (8).

The relationship between tumor-associated anemia and lung cancer prognosis has been the focus of clinicians. A recent meta-analysis of 23 studies that included 10,612 patients showed that preoperative anemia was a prognostic factor of lung cancer (9), as compared with LC patients without anemia, those with pre-operative anemia were at a 1.6 -fold greater risk of death (summarized HR=1.58; 95\% CI, 1.44-1.75). Zhang YH (10) reported that patients with normal pre-treatment hemoglobin (NPHb) levels had a greater chance of surviving for longer period, than did patients with low pre-treatment hemoglobin (LPHb) levels (HR=2.05; 95\% CI, 1.63-2.57; $\mathrm{P}<0.001)$; Mandrekar et al (11) reported that patients with low Hgb levels had significantly worse TTP and OS. Wan et al (12) report that compared to patients with a low $\mathrm{Hb}$ change $(|\Delta \mathrm{Hb}| \leq 2.6)$, those having a $|\Delta \mathrm{Hb}|>2.6$ exhibited a significantly shorter survival [hazard ratio $=1.40,95 \%$ confidence interval 1.31-1.50, $\mathrm{P}=4.5 \times 10(-22)$, $\mathrm{Plog}$ rank=1.6x10(-39)]. Aoe et al reported that anemia is a prognostic factor of patients with lung cancer at first hospital visit (13), survival was significantly shorter in 298 patients with anemia (median survival time (MST): 7.5 months) compared with 313 patients without anemia (MST: 11.8 months, $\mathrm{P}<0.0001$ ). The majority of studies have shown that anemia is a prognostic factor of lung cancer, but controversy still exists; several studies have 
shown that anemia is not associated with the prognosis of lung cancer $(14,15)$. In previous studies on the prognoses of anemia and lung cancer, anemia diagnostic criteria, and detection methods, the results may be biased due to the inconsistency of anemia research subjects. Therefore, in our single-center study, the baseline anemia of 4,874 patients with stage IV non-small cell lung cancer was used as the research object to minimize the interference of related factors.

This study retrospectively analyzed the incidence and grade of baseline anemia in 4,874 patients with stage IV non-small cell lung cancer in China. The relationship between baseline anemia and clinicopathological characteristics and their effects on prognosis were analyzed to further guide the treatment of lung cancer.

\section{Materials and methods}

Clinical data. Our study retrospectively analyzed anemia-related data in patients with stage IV NSCLC. A total of 4,874 patients who were admitted to the Zhejiang Cancer Hospital between January 2013 and June 2019 were eligible for the study. The inclusion criteria were as follows: i) Pathologically proven stage IV NSCLC tumor; ii) age $>18$ years old; and iii) have complete clinical data and follow-up information. The exclusion criteria were as follows: i) With hematological basic diseases; and ii) with chronic nephropathy. The follow-up period lasted until October 2019. Clinical staging was based on the 8th edition of Classification of TNM Lung Cancer (16). Subsequent treatments received by the patient includeradiotherapy, chemotherapy, targeted therapy and immunotherapy, some patients receive only one type of treatment, and some patients receive two or more therapies, combination therapy means that the patient receives two or more treatments. Targeted therapy is defined as targeted drugs corresponding to driver genes of lung cancer. Immunotherapy includes PD1/PD-L1 drug. The study was approved by the institutional review board of Zhejiang Cancer Hospital.

Grading of anemia. According to the anemia grading criteria established by National Cancer Institution (17), anemia is defined as having hemoglobin ( $\mathrm{Hb}$ ) levels of $<120.0 \mathrm{~g} / \mathrm{l}$ for males and $<110.0 \mathrm{~g} / 1$ for females. Anemia is further categorized into five grades based on the $\mathrm{Hb}$ level, as follows: Grade 0, normal Hb level; grade 1 (mild anemia), $100 \mathrm{~g} / 1$, normal level; grade 2 (moderate anemia), 80-100 g/l; grade 3 (severe anemia), 65-80 g/1; and grade 4 (life-threatening anemia), <65 g/l. Baseline anemia is defined as having hemoglobin levels of $<120.0 \mathrm{~g} / 1$ for males and $<110.0 \mathrm{~g} / 1$ for females at first visit to the hospital,and all patients did not receive radiotherapy, chemotherapy, targeted therapy or immunotherapy treatment.

Statistical treatment. The Statistical Package for the Social Sciences (SPSS) version 19.0 (IBM Corp) was used to perform the statistical analysis. Quantitative variables were presented as means \pm standard deviation (mean \pm SD). The mean values from multiple comparisons were compared by using ANOVA followed by Bonferroni-Forsythe. The data was analyzed using $\chi^{2}$. Survival analysis was conducted using the Kaplan-Meier method, and comparison was performed via log-rank test. Multivariate analysis was conducted using Cox proportional hazard regression model. $\mathrm{P}<0.05$ was considered to indicate a statistically significant difference.

\section{Results}

Patient characteristics. A total of 4,874 eligible patients with stage IV NSCLC were included. Patients' sex, age, smoking history, tumor type, bone metastases, comorbidity, and treatment are summarized in Table I.

Prevalence of anemia. For the 4,874 patients who had record of $\mathrm{Hb}$ level, the prevalence of baseline anemia was $32.09 \%$, with a mean $\mathrm{Hb}$ level of $123.32 \pm 20.31 \mathrm{~g} / \mathrm{l}$, of which $19.08 \%$ had mild anemia, $10.79 \%$ had moderate anemia, $1.91 \%$ had severe anemia, and $0.31 \%$ had life-threatening anemia (Table II).

\section{Relationship between baseline anemia and clinical} characteristics. Less female patients had baseline anemia than male patients ( 36.85 vs. $25.56 \%, \mathrm{P}<0.001)$. Compared with younger patients, patients who are $>60$ years old had higher baseline anemia (34.41 vs. $29.31 \%, \mathrm{P}<0.001)$. The prevalence of baseline anemia was highest in patients with squamous cell carcinoma (49.67\%), followed by patients with other NSCLC types (36.93\%). Adenocarcinoma had the lowest prevalence of baseline anemia (29.34\%), $\mathrm{P}<0.001$. Patients with smoking history had higher baseline anemia than nonsmokers (38.97 vs. $27.38 \%, \mathrm{P}<0.001)$. Patients without bone metastases had less baseline anemia than those with bone metastases ( 34.38 vs. $30.11 \%, P=0.002)$. Prevalence of baseline anemia was slightly lower in patients with comorbidity than those without comorbidity (32.06 vs. $32.12 \%$ ). However, the difference was not statistically significant $(\mathrm{P}=0.966)$ (Table III).

Univariate analysis of clinical features and prognosis. Univariate analysis shows the significant association of OS with sex, age, pathological type, bone metastasis, smoking history, chemotherapy, targeted therapy, immunotherapy, baseline anemia, and anemia grade. However, OS was not significantly associated with comorbidity and radiotherapy (Table IV). Patients without baseline anemia had longer OS than those with anemia (median OS: 28.0 vs. 17.4 months, $\mathrm{P}<0.001$; Fig. 1). With rising grade of baseline anemia, OS became shorter. Patients with anemia grade 0 had the longest OS (median OS: 28.0 months), followed by patients with anemia grades 1 and 2 (median OS: 17.5 months). Patients with anemia grades 3 and 4 had the shortest OS (median OS: 8.6 months), $\mathrm{P}<0.001$ (Fig. 2).

Multivariate analysis of clinical features and prognosis. Univariateanalysis of statistically significant sections, such as sex, age, pathological type, bone metastasis, smoking history, chemotherapy, targeted therapy, immunotherapy, baseline anemia, and anemia grade, were included in the COX multivariate regression model. Multivariate analysis showed that age, pathological type, bone metastasis, smoking history, targeted therapy, immunotherapy, baseline anemia, and anemia grade were independent prognostic factors of OS in patients with stage IV NSCLC (Table V). 
Table I. Demographic characteristics of the study population $(n=4,874)$.

\begin{tabular}{|c|c|}
\hline Characteristic & $\mathrm{N}(\%)$ \\
\hline \multicolumn{2}{|l|}{ Sex } \\
\hline Male & $2,822(57.90)$ \\
\hline Female & $2,052(42.10)$ \\
\hline \multicolumn{2}{|l|}{ Age (years) } \\
\hline$>60$ & $2656(54.49)$ \\
\hline$\leq 60$ & $2218(45.51)$ \\
\hline \multicolumn{2}{|l|}{ Smoking history } \\
\hline Yes & $1,981(40.64)$ \\
\hline No & $2,893(59.36)$ \\
\hline \multicolumn{2}{|l|}{ Tumor type } \\
\hline Adenocarcinoma & $3,872(79.44)$ \\
\hline Squamous cell carcinoma & $455(9.34)$ \\
\hline Others NSCLC & $547(11.22)$ \\
\hline \multicolumn{2}{|l|}{ Bone metastasis } \\
\hline Yes & $2,254(46.25)$ \\
\hline No & $2,620(53.75)$ \\
\hline \multicolumn{2}{|l|}{ Comorbidity } \\
\hline Yes & $2,489(51.07)$ \\
\hline No & $2,385(48.93)$ \\
\hline \multicolumn{2}{|l|}{ Radiotherapy } \\
\hline Yes & $1,627(33.38)$ \\
\hline No & $3,247(66.62)$ \\
\hline \multicolumn{2}{|l|}{ Chemotherapy } \\
\hline Yes & $3,426(70.29)$ \\
\hline No & $1,448(29.71)$ \\
\hline \multicolumn{2}{|l|}{ Targeted therapy } \\
\hline Yes & $2,830(58.06)$ \\
\hline No & $2,044(41.94)$ \\
\hline \multicolumn{2}{|l|}{ Immunotherapy } \\
\hline Yes & $204(4.19)$ \\
\hline No & $4,670(95.81)$ \\
\hline \multicolumn{2}{|l|}{ Combine therapy } \\
\hline Yes & $3,093(63.46)$ \\
\hline No & $1,781(36.54)$ \\
\hline
\end{tabular}

NSCLC, non-small cell lung cancer.

\section{Discussion}

Our study included 4,874 patients with stage IV NSCLC. This was the largest sample size of a research on anemia in NSCLC in China. The prevalence of baseline anemia was $32.09 \%$ with a mean $\mathrm{Hb}$ level of $123.32 \pm 20.31 \mathrm{~g} / \mathrm{l}$, of which $19.08 \%$ had mild anemia, $10.79 \%$ had moderate anemia, $1.91 \%$ had severe anemia, and $0.31 \%$ had life-threatening anemia. Most patients with baseline anemia showed mild to moderate anemia. The incidence of baseline anemia was slightly lower than that reported in other countries based on European populations (3) but slightly higher than that reported for American
Table II. Frequency of first admission baseline cancer-related anemia in the current study.

\begin{tabular}{lcrc}
\hline Grade & $\mathrm{Hb}(\text { mean } \pm \mathrm{SD}, \mathrm{g} / \mathrm{l})^{\mathrm{a}}$ & Number & Percentage \\
\hline 0 & $134.02 \pm 13.19$ & 3,310 & - \\
1 & $109.20 \pm 5.72$ & 930 & 19.08 \\
2 & $91.48 \pm 5.61$ & 526 & 10.79 \\
3 & $74.03 \pm 3.97$ & 93 & 1.91 \\
4 & $59.40 \pm 3.94$ & 15 & 0.31 \\
Total & $123.32 \pm 20.31$ & 4,874 & 32.09
\end{tabular}

${ }^{a}$ With significant statistical difference for pairwise comparisons between groups.

populations (6). ECAS is a prospective, observational, and epidemiological survey of the anemia in the European region. At ECAS enrollment, $37.6 \%$ of lung cancer patients had $\mathrm{Hb}$ levels of $<12.0 \mathrm{~g} / \mathrm{dl}$ (3). In a retrospective, observational study on anemia in patients undergoing chemotherapy for solid tumors in the United States, 7,001 patients with NSCLC were enrolled. At baseline, $16.1 \%$ of the patients were anemic with $\mathrm{Hb}$ levels of $<11.0 \mathrm{~g} / \mathrm{dl}$, and $33.6 \%$ had Hb levels of $<12 \mathrm{~g} / \mathrm{dl}$; the mean (SD) $\mathrm{Hb}$ at baseline was 12.7 (1.8) g/dl (6). The difference in results may be related to the different definitions of anemia. Therefore, a standardized and uniform anemia definition may lead to standardized diagnosis and treatment.

Aoe et al reported that in 611 cases of lung cancer in Japan, a significant correlation was found between anemia and age and Eastern Cooperative Oncology Group (ECOG) scores; however, anemia was not correlated with sex, histological type, clinical stage, or serum lactate dehydrogenase (13). The results of our study showed that female patients with baseline anemia were fewer than male patients with baseline anemia (36.85 vs. $25.56 \%, \mathrm{P}<0.001)$. Compared with younger patients, patients who are $>60$ years old had higher prevalence of baseline anemia (34.41 vs. $29.31 \%, \mathrm{P}<0.001)$. The prevalence of baseline anemia was highest in patients with squamous cell carcinoma (49.67\%), followed by patients with other NSCLC types (36.93\%). Patients with adenocarcinoma had the lowest prevalence of baseline anemia $(29.34 \%), \mathrm{P}<0.001$. Our study shows that the proportion of non-smokers is relatively high compared with other study (18), which may be related to the population of the study. A data from the Asian population published by Zhang shows that the proportion of non-smokers is $42.8 \%$ (10), the proportion of non-smoking patients in our study is similar to Zhang's study. In addition, the proportion of female patients in our study is relatively high, while the proportion of female patients who smoke in China is very low; the proportion of patients receiving Epidermal growth factor receptor (EGFR)-tyrosine kinase inhibitor (TKI) targeted therapy in our study is higher, and the dominant population of EGFR-TKI targeted therapy is non-smokers. Patients with smoking history had higher baseline anemia than nonsmokers (38.97 vs. $27.38 \%, \mathrm{P}<0.001$ ). The results were consistent with those obtained in a retrospective study involving 416 NSCLC cases reported by Zhang et al in Henan, China (10). The study further confirmed the basic characteristics of anemia in 
Table III. Relationship between baseline anemia and clinicopathological features in current study.

\begin{tabular}{|c|c|c|c|c|}
\hline Clinical features & Anemia-free group $(n=3,310)$ & Anemia group $(n=1,564)$ & $\chi^{2}$ & P-value \\
\hline Sex & & & 69.829 & $<0.001$ \\
\hline Male & 1782 & 1040 & & \\
\hline Female & 1528 & 524 & & \\
\hline Age (years) & & & 14.466 & $<0.001$ \\
\hline$>60$ & 1742 & 914 & & \\
\hline$\leq 60$ & 1568 & 650 & & \\
\hline Smoking history & & & 72.528 & $<0.001$ \\
\hline Yes & 1209 & 772 & & \\
\hline No & 2101 & 792 & & \\
\hline Pathological type & & & 83.857 & $<0.001$ \\
\hline Adenocarcinoma & 2736 & 1136 & & \\
\hline Squamous cell carcinoma & 229 & 226 & & \\
\hline Others & 345 & 202 & & \\
\hline Bone metastasis & & & 10.132 & 0.002 \\
\hline Yes & 1479 & 775 & & \\
\hline No & 1831 & 789 & & \\
\hline Comorbidity & & & 0.002 & 0.966 \\
\hline Yes & 1691 & 798 & & \\
\hline No & 1619 & 766 & & \\
\hline
\end{tabular}

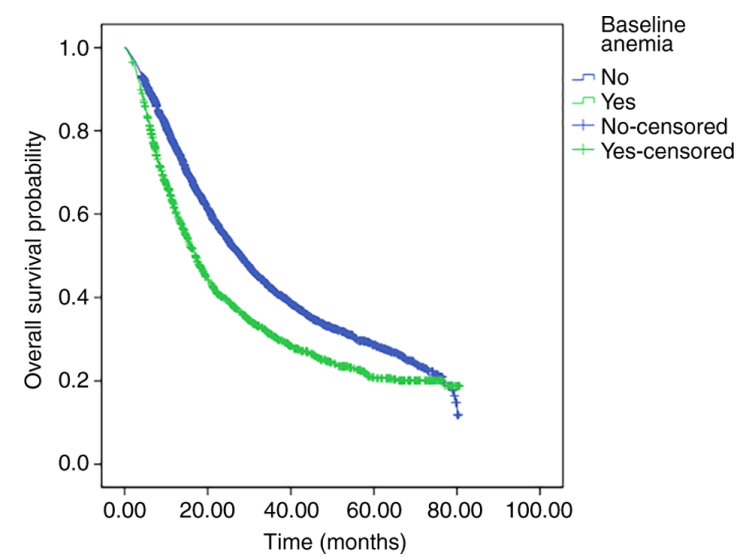

Figure 1. Survival curve of patients with stage IV lung cancer with or without baseline anemia.

Chinese lung cancer patients. The results of our study showed that patients without bone metastases had less baseline anemia than those with bone metastases ( 34.38 vs. $30.11 \%, \mathrm{P}=0.002)$, considering that bone marrow invasion in patients with bone metastasis leads to a decrease in hematopoietic function.

According to previous studies on anemia and lung cancer prognosis, radiotherapy and chemotherapy are the main methods used to treat lung cancer $(10,12,13,18,19)$. In the past decade, targeted therapy and immunotherapy have changed the treatment mode of advanced lung cancer. In our study, the proportions of patients undergoing chemotherapy, targeted therapy, radiotherapy and immunotherapy were 70.29, 58.06, 33.38 and $4.19 \%$, respectively. The proportion of patients undergoing targeted therapy was higher, and immunotherapy

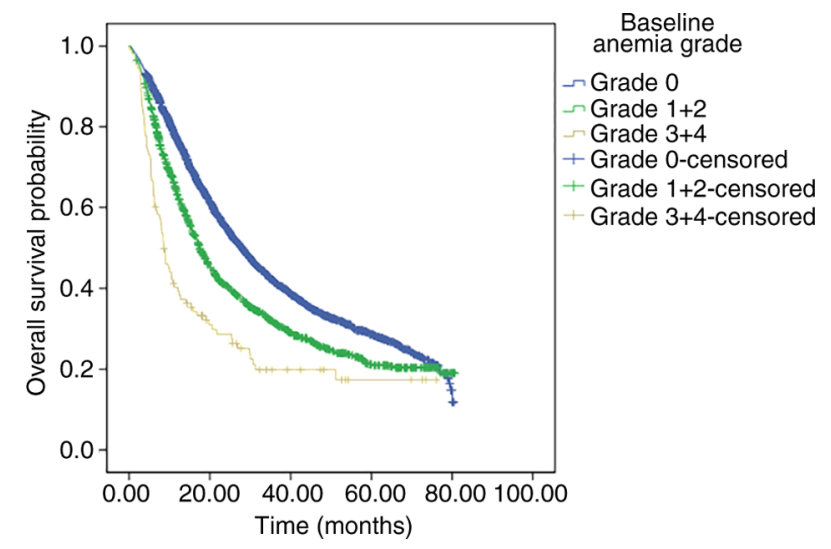

Figure 2. Survival curves of patients with stage IV lung cancer with different anemia grades.

was included in the study; our research reflects the current treatment model for advanced lung cancer (20).

A meta analysis of 23 studies including 10,612 patients showed that preoperative anemia was a prognostic factor of lung cancer (9); patients with preoperative anemia had reduced OS after lung surgery. For patients with advanced lung cancer, the opportunity for surgery is lost; so, the result helps guide patients who will undergo surgery for treatment. Other studies also showed that anemia is associated with the prognosis of lung cancer, but the sample sizes are relatively small or they included multiple cancers (10-13). The evidence from studies investigating the relationship between anemia and the progression of NSCLC remains controversial, as several studies have shown that anemia is not associated with the prognosis of lung 
Table IV. Univariate analysis of clinical features and prognosis in current study.

\begin{tabular}{|c|c|c|c|c|}
\hline Clinical features & OS (months) & $95 \% \mathrm{CI}$ & $\chi^{2}$ & P-value \\
\hline Sex & & & 74.224 & $<0.001$ \\
\hline Male & 19.800 & $18.574-21.026$ & & \\
\hline Female & 30.800 & $28.523-33.077$ & & \\
\hline Age (years) & & & 52.673 & $<0.001$ \\
\hline$>60$ & 20.400 & $19.198-21.602$ & & \\
\hline$\leq 60$ & 29.400 & $27.344-31.456$ & & \\
\hline Smoking history & & & 90.913 & $<0.001$ \\
\hline Yes & 18.300 & $17.087-19.513$ & & \\
\hline No & 29.200 & 27.296-31.104 & & \\
\hline Pathological type & & & 88.626 & $<0.001$ \\
\hline Adenocarcinoma & 26.900 & $25.575-28.225$ & & \\
\hline Squamous cell carcinoma & 15.300 & $13.554-17.046$ & & \\
\hline Others & 13.800 & $11.950-15.650$ & & \\
\hline Bone metastasis & & & 32.394 & $<0.001$ \\
\hline Yes & 21.100 & $19.693-22.507$ & & \\
\hline No & 27.100 & $25.323-28.877$ & & \\
\hline Comorbidity & & & 0.729 & 0.393 \\
\hline Yes & 22.800 & $21.067-24.533$ & & \\
\hline No & 25.100 & $23.499-26.701$ & & \\
\hline Radiotherapy & & & 2.030 & 0.154 \\
\hline Yes & 25.200 & $23.030-27.370$ & & \\
\hline No & 23.400 & $21.917-24.883$ & & \\
\hline Chemotherapy & & & 8.371 & 0.004 \\
\hline Yes & 23.100 & $21.758-24.442$ & & \\
\hline No & 26.400 & $23.422-29.378$ & & \\
\hline Targeted therapy & & & 182.633 & $<0.001$ \\
\hline Yes & 30.500 & $28.796-32.204$ & & \\
\hline No & 15.700 & $14.629-16.771$ & & \\
\hline Immunotherapy & & & 6.174 & 0.013 \\
\hline Yes & 29.400 & $26.369-32.431$ & & \\
\hline No & 23.800 & $22.599-25.001$ & & \\
\hline Anemia & & & 84.801 & $<0.001$ \\
\hline No $(n=3,382)$ & 28.000 & $26.480-29.520$ & & \\
\hline Yes $(n=1,596)$ & 17.400 & $15.887-18.313$ & & \\
\hline Grade of anemia & & & 105.379 & $<0.001$ \\
\hline $0(\mathrm{n}=3,382)$ & 28.000 & $26.480-29.520$ & & \\
\hline $1+2(\mathrm{n}=1,146)$ & 17.500 & $16.190-18.810$ & & \\
\hline $3+4(n=110)$ & 8.600 & $6.857-10.343$ & & \\
\hline
\end{tabular}

OS, overall survival; CI, confidence interval.

cancer $(14,15)$. Our study is a single-center study with a large sample size that was performed in the real world in China. We analyzed the relationship between baseline anemia and prognosis in 4,874 patients with IV non-small cell lung cancer. Univariate analysis results showed that patients without baseline anemia had longer OS than those with anemia (median OS: 28.0 vs. 17.4 months, $\mathrm{P}<0.001$ ). Further multivariate analysis revealed that baseline anemia was an independent prognostic factor in patients with stage IV NSCLC. Our conclusion is consistent with the relevant research results (9-13). The reason for the shortened survival of patients due to tumor-associated anemia is the decreased sensitivity to treatment and tolerance to treatment. According to the anemia grade, we divided the patients into three groups, namely, anemia grades $0,1-2$, and 3-4. With rising anemia grade, the OS became shorter. The patients with anemia grade 0 had the longest OS (median OS: 28.0 months), followed by patients with anemia grades 1 and 2 (median OS: 17.5 months). The patients with anemia grades 3 
Table V. Multivariate analysis of clinical features and prognosis in current study.

\begin{tabular}{|c|c|c|c|c|c|c|}
\hline Clinical features & $\mathrm{B}$ & SE & Wald & HR & $95 \% \mathrm{CI}$ & P-value \\
\hline Sex & -0.068 & 0.053 & 1.655 & 0.934 & $0.842-1.036$ & 0.198 \\
\hline Age & 0.183 & 0.039 & 22.420 & 1.201 & $1.113-1.296$ & $<0.001$ \\
\hline Pathological type & 0.151 & 0.028 & 28.567 & 1.163 & $1.100-1.229$ & $<0.001$ \\
\hline Bone metastasis & -0.261 & 0.038 & 47.710 & 0.770 & $0.715-0.829$ & $<0.001$ \\
\hline Smoking history & -0.160 & 0.052 & 9.481 & 0.852 & $0.7700-0.944$ & 0.002 \\
\hline Targeted therapy & 0.415 & 0.040 & 108.417 & 1.514 & $1.401-1.637$ & $<0.001$ \\
\hline Chemotherapy & -0.008 & 0.046 & 0.029 & 0.992 & $0.907-1.085$ & 0.865 \\
\hline Immunotherapy & 0.406 & 0.111 & 13.281 & 1.501 & $1.206-1.867$ & $<0.001$ \\
\hline Baseline anemia & -0.280 & 0.130 & 4.638 & 0.756 & $0.586-0.975$ & 0.031 \\
\hline Grade of anemia & 0.489 & 0.115 & 18.213 & 1.630 & $1.303-2.041$ & $<0.001$ \\
\hline
\end{tabular}

SE, standard error; HR, hazard ratio; CI, confidence interval.

and 4 had the shortest OS (median OS: 8.6 months), $\mathrm{P}<0.001$. Multivariate analysis showed that baseline anemia grade was also a prognostic factor of stage IV NSCLC patients. Results further confirmed the importance of baseline anemia for NSCLC, which requires attention and timely treatment (21). Treatment of tumor-associated anemia include the correction of nutritional deficiencies, use of intravenous (IV) iron, erythropoietic stimulating agents (ESAs), and blood transfusions (2), the use of ESAs is still controversial due to its security issues. The results of a phase III clinical study on ESAs treatment showed that Darbepoetinalfa dosed to a $12.0-\mathrm{g} / \mathrm{dl} \mathrm{Hb}$ ceiling was noninferior to placebo for OS and PFS and significantly reduced odds of transfusion or $\mathrm{Hb} \leq 8.0 \mathrm{~g} / \mathrm{dl}$ in anemic patients with NSCLC receiving myelosuppressive chemotherapy (21). The early treatment of anemia is very important. Whether management of anemia can improve the survival of patients, it may be an aim of future studies.

Our study had some limitations. The major limitation is its retrospective nature. Moreover, the study is a single-center study. However, as the largest sample size research on anemia in NSCLC, our results are meaningful and can serve as a guide for the treatment of NSCLC patients with anemia in China. In the future, multi-center prospective studies are needed.

The study showed that the prevalence of baseline anemia was highest in patients with stage IV NSCLC who are men, $>60$ years old, with smoking history, with squamous cell carcinoma, and with bone metastases. Baseline anemia and anemia grading were independent prognostic factors in patients with stage IV NSCLC.

\section{Acknowledgments}

Not applicable.

\section{Funding}

No funding was received.

\section{Availability of data and materials}

Not applicable.

\section{Authors' contributions}

JZ designed the study. CC and ZS conceived of the study. $\mathrm{CC}$ and $\mathrm{WW}$ acquired the data. $\mathrm{CC}$ and $\mathrm{ZS}$ performed data analysis/interpretation. CC performed statistical analysis. $\mathrm{CC}$ prepared the manuscript. JZ revised the manuscript. The authenticity of all the raw data is confirmed by CC and JZ. All authors read and approved the final manuscript.

\section{Ethics approval and consent to participate}

The current study was approved by the institutional review board of Zhejiang Cancer Hospital.

\section{Patient consent for publication}

Not applicable.

\section{Competing interests}

The authors declare that they have no competing interests.

\section{References}

1. Chen W, Sun K, Zheng R, Zeng H, Zhang S, Xia C, Yang Z, Li H, Zou X and He J: Cancer incidence and mortality in China, 2014. Chin J Cancer Res 30: 1-12, 2018.

2. Gilreath JA, Stenehjem DD and Rodgers GM: Diagnosis and treatment of cancer-related anemia. Am J Hematol 89: 203-212, 2014.

3. Ludwig H, Van Belle S, Barrett-Lee P, Birgegård G, Bokemeyer C, Gascón P, Kosmidis P, Krzakowski M, Nortier J, Olmi P, et al: The European Cancer Anaemia Survey (ECAS): A large, multinational, prospective survey defining the prevalence, incidence, and treatment of anaemia in cancer patients. Eur J Cancer 40: 2293-2306, 2004.

4. Crawford J, Kosmidis PA, Hirsch FR and Langer CJ: Targeting anemia in patients with lung cancer. J Thorac Oncol 1: 716-725, 2006.

5. Gascón P, Almenárez J, Artal Á, Camps C, Fírvida JL, Garrido P, González Larriba JL and Montalar J: Management of lung cancer-associated anaemia: The spanish lung cancer anaemia survey (SLCAS). Clin Transl Oncol 13: 328-334, 2011.

6. Wu Y, Aravind S, Ranganathan G, Martin A and Nalysnyk L: Anemia and thrombocytopenia in patients undergoing chemotherapy for solid tumors: A descriptive study of a large outpatient oncology practice database, 2000-2007. Clin Ther 31: 2416-2432, 2009. 
7. Muñoz-Langa J, De Castro J, Gascó P, Sanchez A, Esteban E, Gasent JM, Barneto I, Montalar J, Artal A and Vidal S: Chemotherapy-associated anemia in patients with lung cancer: An epidemiological, retrospective and multicenter study. Future Oncol 11: 1665-1674, 2015.

8. Cella D, Kallich J, McDermott A and Xu X: The longitudinal relationship of hemoglobin, fatigue and quality of life in anemic cancer patients: Results from five randomized clinical trials. Ann Oncol 15: 979-986, 2004.

9. Liu Y, Bai YP, Zhou ZF, Jiang CR, Xu Z and Fan XX: Preoperative anemia as a prognostic factor in patients with lung cancer: A systematic review and meta-analysis of epidemiological studies. J Cancer 10: 2047-2056, 2019.

10. Zhang YH, Lu Y, Lu H, Zhang MW, Zhou YM, Li XL, Lv P and Zhao XY: Pre-treatment hemoglobin levels are an independent prognostic factor in patients with non-small cell lung cancer. Mol Clin Oncol 9: 44-49, 2018.

11. Mandrekar SJ, Schild SE, Hillman SL, Allen KL, Marks RS, Mailliard JA, Krook JE, Maksymiuk AW, Chansky K, Kelly K, et al: A prognostic model for advanced stage nonsmall cell lung cancer. Pooled analysis of North Central Cancer Treatment Group trials. Cancer 107: 781-792, 2006.

12. Wan S, Lai Y, Myers RE, Li B, Palazzo JP, Burkart AL, Chen G, Xing $\mathrm{J}$ and Yang H: Post-diagnosis hemoglobin change associates with overall survival of multiple malignancies-results from a 14-year hospital-based cohort of lung, breast, colorectal, and liver cancers. BMC Cancer 13: 340, 2013.

13. Aoe K, Hiraki A, Maeda T, Katayama H, Fujiwara K, Tabata M, Kiura K, Ueoka H and Tanimoto M: Serum hemoglobin level determined at the first presentation is a poor prognostic indicator in patients with lung cancer. Intern Med 44: 800-804, 2005.

14. Gauthier I, Ding K, Winton T, Shepherd FA, Livingston R, Johnson DH, Rigas JR, Whitehead M, Graham B and Seymour L: Impact of hemoglobin levels on outcomes of adjuvant chemotherapy in resected non-small cell lung cancer: The JBR.10 trial experience. Lung Cancer 55: 357-363, 2007.
15. Trufelli DC, Moraes TV, Lima AA and Giglio AD: Epidemiological profile and prognostic factors in patients with lung cancer. Rev Assoc Med Bras (1992) 62: 428-433, 2016.

16. Kay FU, Kandathil A, Batra K, Saboo SS, Abbara S and Rajiah P: Revisions to the Tumor, Node, Metastasis staging of lung cancer (8th edition): Rationale, radiologic findings and clinical implications. World J Radiol 9: 269-279, 2017.

17. Experts Committee on Cancer-Related Anemia and Chinese Society of Clinical Oncology (CSCO): Clinical practice guidelines on cancer-related anemia (2012-2013 Edition). Chin Clin Oncol 1: 18, 2012.

18. Pathak RS, Pantarotto JR, Cook G, Holmes O, Cross P and MacRae RM: Anemia is a poor prognostic factor for stage I non-small cell lung cancer (NSCLC) patients treated with Stereotactic Body Radiation Therapy (SBRT). Clin Transl Radiat Oncol 16: 28-33, 2019.

19. Topkan E, Selek U, Ozdemir Y, Yildirim BA, Guler OC, Mertsoylu $\mathrm{H}$ and Hahn SM: Chemoradiotherapy-induced hemoglobin nadir values and survival in patients with stage III non-small cell lung cancer. Lung Cancer 121: 30-36, 2018.

20. Passiglia F, Pilotto S, Facchinetti F, Bertolaccini L, Del Re M, Ferrara R, Franchina T, Malapelle U, Menis J, Passaro A, et al: Treatment of advanced non-small-cell lung cancer: The 2019 AIOM (Italian Association of Medical Oncology) clinical practice guidelines. Crit Rev Oncol Hematol 146: 102858, 2020.

21. Gascón P, Nagarkar R, Šmakal M, Syrigos KN, Barrios CH, Sánchez JC, Zhang L, Henry DH, Gordon D, Hirsh V, et al: A randomized, double-blind, placebo-controlled, phase III noninferiority study of the long-term safety and efficacy of darbepoetinalfa for chemotherapy-induced anemia in patients with advanced NSCLC. J Thorac Oncol 15: 190-202, 2020.

This work is licensed under a Creative Commons Attribution-NonCommercial-NoDerivatives 4.0 International (CC BY-NC-ND 4.0) License. 